\title{
On the Dynamics of the Higher Order Nonlinear Rational Difference Equation
}

\author{
M. A. El-Moneam* \\ Mathematics Department, Faculty of Science and Arts in Farasan, Jazan University, Kingdom of Saudi Arabia
}

Received: 26 Nov. 2013, Revised: 3 Mar. 2014, Accepted: 6 Mar. 2014

Published online: 1 May 2014

\begin{abstract}
In this article, we study the periodicity, the boundedness and the global stability of the positive solutions of the following nonlinear difference equation

$$
x_{n+1}=A x_{n}+B x_{n-k}+C x_{n-l}+D x_{n-\sigma}+\frac{b x_{n-k}}{\left[d x_{n-k}-e x_{n-l}\right]}, \quad n=0,1,2, \ldots \ldots
$$

where the coefficients $A, B, C, D, b, d, e \in(0, \infty)$, while $k, l$ and $\sigma$ are positive integers. The initial conditions $x_{-\sigma}, \ldots, x_{-l}, \ldots, x_{-k}, \ldots, x_{-1}, x_{0}$ are arbitrary positive real numbers such that $k<l<\sigma$. Some numerical examples will be given to illustrate our results.
\end{abstract}

Keywords: Difference equations, prime period two solution, boundedness character, locally asymptotically stable, global attractor, global stability.

\section{Introduction}

The topic of difference equations is interesting and attractive to many mathematicians working in this field. The qualitative study of difference equations is a fertile research area and increasingly attracts many mathematicians. This topic draws its importance from the fact that many real life phenomena are modeled using difference equations. Examples from economy, biology, etc. can be found in $[12,17,18]$. It is known that nonlinear difference equations are capable of producing a complicated behavior regardless its order. This can be easily seen from the family $x_{n+1}=g_{\mu}\left(x_{n}\right), \mu>0, n \geq 0$. This behavior is ranging according to the value of $\mu$, from the existence of a bounded number of periodic solutions to chaos.

There has been a great interest in studying the global attractivity, the boundedness character and the periodicity nature of nonlinear difference equations. For example, in the articles [13-16, 19-26] closely related global convergence results were obtained which can be applied to nonlinear difference equations in proving that every solution of these equations converges to a period two solution. For other closely related results, (see [2]-[11],[28]-[31]) and the references cited therein. The study of these equations is challenging and rewarding and is still in its infancy. We believe that the nonlinear rational difference equations are of paramount importance in their own right. Furthermore the results about such equations offer prototypes for the development of the basic theory of the global behavior of nonlinear difference equations.

The objective of this article is to investigate some qualitative behavior of the solutions of the nonlinear difference equation

$$
\begin{aligned}
x_{n+1} & =A x_{n}+B x_{n-k}+C x_{n-l}+D x_{n-\sigma}+\frac{b x_{n-k}}{\left[d x_{n-k}-e x_{n-l}\right]}, \\
n & =0,1,2, \ldots .
\end{aligned}
$$

where the coefficients $A, B, C, D, b, d, e \in(0, \infty)$, while $k, l$ and $\sigma$ are positive integers. The initial conditions $x_{-\sigma}, \ldots, x_{-l}, \ldots, x_{-k}, \ldots, x_{-1}, x_{0}$ are arbitrary positive real numbers such that $k<l<\sigma$. Note that the special cases of Eq.(1) have been studied in [1] when $B=C=D=0$, and $k=0, l=1, b$ is replaced by $-b$ and in [27] when $B=C=D=0$, and $k=0, b$ is replaced by $-b$ and in [33] when $B=C=D=0, l=0$ and in [32] when $A=C=D=0, l=0, b$ is replaced by $-b$.

\footnotetext{
* Corresponding author e-mail: mabdelmeneam2014@yahoo.com
} 
Our interest now is to study behavior of the solutions of Eq.(1) in its general form. For the related work, (see [34]-[39]). Let us now recall some well known results [12] which will be useful in the sequel.

Definition 1.Consider a difference equation in the form

$$
x_{n+1}=F\left(x_{n}, x_{n-k}, x_{n-l}, x_{n-\sigma}\right), \quad n=0,1,2, \ldots \ldots
$$

where $F$ is a continuous function, while $k$ and $l$ are positive integers such that $k<l<\sigma$. An equilibrium point $\widetilde{x}$ of this equation is a point that satisfies the condition $\widetilde{x}=F(\widetilde{x}, \widetilde{x}, \widetilde{x}, \widetilde{x})$. That is, the constant sequence $\left\{x_{n}\right\}$ with $x_{n}=\tilde{x}$ for all $n \geq-k \geq-l \geq \sigma$ is a solution of that equation.

Definition 2.Let $\tilde{x} \in(0, \infty)$ be an equilibrium point of Eq.(2). Then we have

(i) An equilibrium point $\tilde{x}$ of Eq.(2) is called locally stable if for every $\varepsilon>0$ there exists $\delta>0$ such that, if $x_{-\sigma}$, $\ldots, x_{-l}, \ldots, x_{-k}, \ldots, x_{-1}, x_{0} \in(0, \infty)$ with $\left|x_{-\sigma}-\tilde{x}\right|+\ldots+$ $\left|x_{-l}-\tilde{x}\right|+\ldots+\left|x_{-k}-\widetilde{x}\right|+\ldots+\left|x_{-1}-\tilde{x}\right|+\left|x_{0}-\widetilde{x}\right|<\delta$, then $\left|x_{n}-\tilde{x}\right|<\varepsilon$ for all $n \geq-k \geq-l$.

(ii) An equilibrium point $\tilde{x}$ of Eq.(2) is called locally asymptotically stable if it is locally stable and there exists $\gamma>0$ such that, if $x_{-\sigma}, \ldots, x_{-l}, \ldots, x_{-k}, \ldots, x_{-1}, x_{0} \in(0, \infty)$ with $\left|x_{-\sigma}-\widetilde{x}\right|+\ldots+\left|x_{-l}-\tilde{x}\right|+\ldots+\left|x_{-k}-\tilde{x}\right|+\ldots+$ $\left|x_{-1}-\tilde{x}\right|+\left|x_{0}-\tilde{x}\right|<\gamma$, then

$$
\lim _{n \rightarrow \infty} x_{n}=\tilde{x}
$$

(iii) An equilibrium point $\widetilde{x}$ of Eq.(2) is called a global attractor iffor every $x_{-\sigma}, \ldots, x_{-l}, \ldots, x_{-k}, \ldots, x_{-1}, x_{0} \in(0, \infty)$ we have

$$
\lim _{n \rightarrow \infty} x_{n}=\tilde{x}
$$

(iv) An equilibrium point $\tilde{x}$ of Eq.(2) is called globally asymptotically stable if it is locally stable and a global attractor.

(v) An equilibrium point $\widetilde{x}$ of Eq.(2) is called unstable if it is not locally stable.

Definition 3.A sequence $\left\{x_{n}\right\}_{n=-\sigma}^{\infty}$ is said to be periodic with period $r$ if $x_{n+r}=x_{n}$ for all $n \geq-\sigma$. A sequence $\left\{x_{n}\right\}_{n=-\sigma}^{\infty}$ is said to be periodic with prime period $r$ if $r$ is the smallest positive integer having this property.

Definition 4.Eq.(2) is called permanent and bounded if there exists numbers $m$ and $M$ with $0<m<M<\infty$ such that for any initial conditions $x_{-\sigma}, \ldots, x_{-l}, \ldots, x_{-k}, \ldots, x_{-1}$, $x_{0} \in(0, \infty)$ there exists a positive integer $N$ which depends on these initial conditions such that

$$
m \leq x_{n} \leq M \text { for all } n \geq N
$$

Definition 5.The linearized equation of Eq.(2) about the equilibrium point $\tilde{x}$ is defined by the equation

$$
z_{n+1}=\rho_{0} z_{n}+\rho_{1} z_{n-k}+\rho_{2} z_{n-l}+\rho_{3} z_{n-\sigma}=0,
$$

where

$$
\begin{aligned}
\rho_{0} & =\frac{\partial F(\tilde{x}, \tilde{x}, \tilde{x}, \tilde{x})}{\partial x_{n}}, \rho_{1}=\frac{\partial F(\widetilde{x}, \tilde{x}, \tilde{x}, \tilde{x})}{\partial x_{n-k}}, \rho_{2}=\frac{\partial F(\tilde{x}, \tilde{x}, \tilde{x}, \tilde{x})}{\partial x_{n-l}}, \\
\rho_{3} & =\frac{\partial F(\tilde{x}, \tilde{x}, \tilde{x}, \tilde{x})}{\partial x_{n-\sigma}} .
\end{aligned}
$$

The characteristic equation associated with Eq.(3) is

$$
\rho(\lambda)=\lambda^{\sigma+1}-\rho_{0} \lambda^{\sigma}-\rho_{1} \lambda^{\sigma-k}-\rho_{2} \lambda^{\sigma-l}-\rho_{3}=0 .
$$

Theorem 1.[12]. Assume that $F$ is a $C^{1}-$ function and let $\tilde{x}$ be an equilibrium point of Eq.(2). Then the following statements are true.

(i) If all roots of Eq.(4) lie in the open unit disk $|\lambda|<1$, then the equilibrium point $\widetilde{x}$ is locally asymptotically stable.

(ii) If at least one root of Eq.(4) has absolute value greater than one, then the equilibrium point $\widetilde{x}$ is unstable.

(iii) If all roots of Eq.(4) have absolute value greater than one, then the equilibrium point $\widetilde{x}$ is a source.

Theorem 2.[18]. Assume that $\rho_{0}, \rho_{1}, \rho_{2}$ and $\rho_{3} \in R$. Then

$$
\left|\rho_{0}\right|+\left|\rho_{1}\right|+\left|\rho_{2}\right|+\left|\rho_{3}\right|<1
$$

is a sufficient condition for the asymptotic stability of Eq.(2).

Theorem 3.[12]. Consider the difference equation (2). Let $\widetilde{x} \in I$ be an equilibrium point of Eq.(2). Suppose also that

(i) $F$ is a nondecreasing function in each of its arguments.

(ii) The function $F$ satisfies the negative feedback property

$$
[F(x, x, x, x)-x](x-\tilde{x})<0 \quad \text { for all } x \in I-\{\widetilde{x}\},
$$

where $I$ is an open interval of real numbers. Then $\tilde{x}$ is global attractor for all solutions of Eq.(2).

\section{The local stability of the solutions}

The equilibrium point $\widetilde{x}$ of Eq.(1) is the positive solution of the equation

$$
\widetilde{x}=(A+B+C+D) \widetilde{x}+\frac{b \widetilde{x}}{(d-e) \widetilde{x}},
$$


where $d \neq e$. If $[(A+B+C+D)-1](e-d)>0$, then the only positive equilibrium point $\widetilde{x}$ of Eq.(1) is given by

$$
\widetilde{x}=\frac{b}{[(A+B+C+D)-1](e-d)} .
$$

Let us now introduce a continuous function $F:(0, \infty)^{4} \longrightarrow(0, \infty)$ which is defined by

$F\left(u_{0}, u_{1}, u_{2}, u_{3}\right)=A u_{0}+B u_{1}+C u_{2}+D u_{3}+\frac{b u_{1}}{\left(d u_{1}-e u_{2}\right)}$,

provided $d u_{1} \neq e u_{2}$. Consequently, we get

$$
\left\{\begin{array}{c}
\frac{\partial F(\widetilde{x}, \widetilde{x}, \widetilde{x})}{\partial u_{0}}=A=\rho_{0} \\
\frac{\partial F(\widetilde{x}, \widetilde{x}, \widetilde{x})}{\partial u_{1}}=B-\frac{e[(A+B+C)-1]}{(e-d)}=\rho_{1} \\
\frac{\partial F(\widetilde{x}, \widetilde{x}, \tilde{x})}{\partial u_{2}}=C+\frac{e[(A+B+C)-1]}{(e-d)}=\rho_{2} \\
\frac{\partial F(\widetilde{x}, \widetilde{x}, \widetilde{x})}{\partial u_{3}}=D=\rho_{3}
\end{array}\right.
$$

where $e \neq d$. Thus, the linearized equation of Eq.(1) about $\tilde{x}$ takes the form

$$
z_{n+1}-\rho_{0} z_{n}-\rho_{1} z_{n-k}-\rho_{2} z_{n-l}-\rho_{3} z_{n-\sigma}=0,
$$

where $\rho_{0}, \rho_{1}, \rho_{2}$ and $\rho_{3}$ are given by (9).

Theorem 4.Assume that $e \neq d, A+B+C+D \neq 1$ and

$$
|A(e-d)|+|B(e-d)-e[(A+B+C+D)-1]|+
$$

$|C(e-d)+e[(A+B+C+D)-1]|+|D(e-d)|<|e-d|$,

then the equilibrium point (7) of Eq.(1) is locally asymptotically stable.

Proof.From (9) and (11) we deduce that $\left|\rho_{0}\right|+\left|\rho_{1}\right|+\left|\rho_{2}\right|+\left|\rho_{3}\right|<1$, and hence the proof follows with the aid of Theorem 2 .

\section{Periodic solutions}

In this section, we study the existence of periodic solutions of Eq.(1). The following theorem states the necessary and sufficient conditions that the equation has periodic solutions of prime period two.

Theorem 5.If $k, l$ and $\sigma$ are both even positive integers, then Eq.(1) has no prime period two solution.

Proof.Assume that there exists distinct positive solutions $P, Q, P, Q, \ldots \ldots$ of prime period two of Eq.(1). If $k, l$ and $\sigma$ are both even positive integers, then $x_{n}=x_{n-k}=x_{n-l}=x_{n-\sigma}$. It follows from Eq.(1) that

$$
P=(A+B+C+D) Q-\frac{b}{(e-d)},
$$

and

$$
Q=(A+B+C+D) P-\frac{b}{(e-d)} .
$$

By subtracting (13) from (12), we get

$$
(P-Q)[A+B+C+D+1]=0 .
$$

Since $A+B+C+D+1 \neq 0$, then $P=Q$. This is a contradiction. Thus, the proof is now completed. $\square$

Theorem 6.If $k, l$ and $\sigma$ are both odd positive integers and $A+1 \neq B+C+D$, then Eq.(1) has no prime period two solution.

Proof.Following the proof of Theorem 5, we deduce that if $k, l$ and $\sigma$ are both odd positive integers, then $x_{n+1}=$ $x_{n-k}=x_{n-l}=x_{n-\sigma}$. It follows from Eq.(1) that

$$
P=A Q+(B+C+D) P-\frac{b}{(e-d)},
$$

and

$$
Q=A P+(B+C+D) Q-\frac{b}{(e-d)} .
$$

By subtracting (15) from (14), we get

$$
(P-Q)[(A+1)-(B+C+D)]=0 .
$$

Since $(A+1)-(B+C+D) \neq 0$, then $P=Q$. This is a contradiction. Thus, the proof is now completed.

Theorem 7.If $k, l$ are even and $\sigma$ is odd positive integers and $(A+B+C)+1 \neq D$, then Eq.(1) has no prime period two solution.

Proof.Following the proof of Theorem 5, we deduce that if $k, l$ are even and $\sigma$ is odd positive integers, then $x_{n}=$ $x_{n-k}=x_{n-l}$ and $x_{n+1}=x_{n-\sigma}$. It follows from Eq.(1) that

$$
P=(A+B+C) Q+D P-\frac{b}{(e-d)},
$$

and

$$
Q=(A+B+C) P+D Q-\frac{b}{(e-d)} .
$$

By subtracting (17) from (16), we get

$$
(P-Q)[(A+B+C+1)-D]=0,
$$

Since $(A+B+C+1)-D \neq 0$, then $P=Q$. This is a contradiction. Thus, the proof is now completed. $\square$ 
Theorem 8.If $\sigma$ is even and $k, l$ are odd positive integers and $(A+D+1) \neq(B+C)$, then Eq.(1) has no prime period two solution.

Proof.Following the proof of Theorem 5, we deduce that if $\sigma$ is even and $k, l$ are odd positive integers, then $x_{n}=x_{n-\sigma}$ and $x_{n+1}=x_{n-k}=x_{n-l}$. It follows from Eq.(1) that

$$
P=(A+D) Q+(B+C) P-\frac{b}{(e-d)},
$$

and

$$
Q=(A+D) P+(B+C) Q-\frac{b}{(e-d)} .
$$

By subtracting (19) from (18), we get

$$
(P-Q)[(A+D+1)-(B+C)]=0,
$$

Since $(A+D+1)-(B+C) \neq 0$, then $P=Q$. This is a contradiction. Thus, the proof is now completed. $\square$

Theorem 9.If $k$ is even and $l, \sigma$ are odd positive integers, then Eq.(1) has prime period two solution if the condition

$$
(1-(C+D))(3 e-d)<(e+d)(A+B),
$$

is valid, provided $(C+D)<1$ and $e(1-(C+D))-d(A+B)>0$.

Proof.If $k$ is even and $l, \sigma$ are odd positive integers, then $x_{n}=x_{n-k}$ and $x_{n+1}=x_{n-l}=x_{n-\sigma}$. It follows from Eq.(1) that

$$
P=(A+B) Q+(C+D) P-\frac{b Q}{(e P-d Q)},
$$

and

$$
Q=(A+B) P+(C+D) Q-\frac{b P}{(e Q-d P)} .
$$

Consequently, we get

$$
\begin{aligned}
e P^{2}-d P Q= & e(A+B) P Q-d(A+B) Q^{2}+e(C+D) P^{2} \\
& -(C+D) d P Q-b Q,
\end{aligned}
$$

and

$$
\begin{aligned}
e Q^{2}-d P Q= & e(A+B) P Q-d(A+B) P^{2}+e(C+D) Q^{2} \\
& -(C+D) d P Q-b P .
\end{aligned}
$$

By subtracting (24) from (23), we get

$$
P+Q=\frac{b}{[e(1-(C+D))-d(A+B)]},
$$

where $e(1-(C+D))-d(A+B)>0$. By adding (23) and (24), we obtain

$$
P Q=\frac{e b^{2}(1-(C+D))}{(e+d)\left[K_{1}+(A+B)\right]\left[e K_{1}-d(A+B)\right]^{2}},
$$

where $K_{1}=(1-(C+D))$, provided $(C+D)<1$. Assume that $P$ and $Q$ are two positive distinct real roots of the quadratic equation

$$
t^{2}-(P+Q) t+P Q=0 .
$$

Thus, we deduce that

$$
(P+Q)^{2}>4 P Q
$$

Substituting (25) and (26) into (28), we get the condition (20). Thus, the proof is now completed. $\square$

Theorem 10.If $l$ is even and $k, \sigma$ are odd positive integers, then Eq.(1) has prime period two solution if the condition

$$
(A+C)(3 e-d)<(e+d)(1-(B+D)),
$$

is valid, provided $(B+D)<1$ and $d(1-(B+D))-e(A+C)>0$.

Proof.If $l$ is even and $k, \sigma$ are odd positive integers, then $x_{n}=x_{n-l}$ and $x_{n+1}=x_{n-k}=x_{n-\sigma}$. It follows from Eq.(1) that

$$
P=(A+C) Q+(B+D) P-\frac{b P}{(e Q-d P)},
$$

and

$$
Q=(A+C) P+(B+D) Q-\frac{b Q}{(e P-d Q)} .
$$

Consequently, we get

$$
P+Q=\frac{b}{[d(1-(B+D))-e(A+C)]},
$$

where $d(1-(B+D))-e(A+C)>0$,

$$
P Q=\frac{e b^{2}(A+C)}{(e+d)\left[K_{2}+(A+C)\right]\left[d K_{2}-e(A+C)\right]^{2}},
$$

where $K_{2}=(1-(B+D))$, provided $(B+D)<1$. Substituting (32) and (33) into (28), we get the condition (29). Thus, the proof is now completed. $\square$

Theorem 11.If $l, \sigma$ are even and $k$ is odd positive integers, then Eq.(1) has prime period two solution if the condition

$$
(A+C+D)(3 e-d)<(e+d)(1-B),
$$

is valid, provided $B<1$ and $d(1-B)-e(A+C+D)>$ 0 .

Proof.If $l, \sigma$ are even and $k$ is odd positive integers, then $x_{n}=x_{n-l}=x_{n-\sigma}$ and $x_{n+1}=x_{n-k}$. It follows from Eq.(1) that

$$
P=(A+C+D) Q+B P-\frac{b P}{(e Q-d P)},
$$


and

$$
Q=(A+C+D) P+B Q-\frac{b Q}{(e P-d Q)} .
$$

Consequently, we get

$$
P+Q=\frac{b}{[d(1-B)-e(A+C+D)]},
$$

where $d(1-B)-e(A+C+D)>0$,

$$
P Q=\frac{e b^{2}(A+C+D)}{(e+d)\left[(1-B)+K_{3}\right]\left[d(1-B)-e K_{3}\right]^{2}},
$$

where $K_{3}=(A+C+D)$, provided $B<1$. Substituting (37) and (38) into (28), we get the condition (34). Thus, the proof is now completed.

Theorem 12.If $k, \sigma$ are even and $l$ is odd positive integers, then Eq.(1) has prime period two solution if the condition

$$
(3 e-d)(1-C)<(e+d)(A+B+D),
$$

is valid, provided $C<1$ and $e(1-C)-d(A+B+D)>$ 0 .

Proof.If $k, \sigma$ are even and $l$ is odd positive integers, then $x_{n}=x_{n-k}=x_{n-\sigma}$ and $x_{n+1}=x_{n-l}$. It follows from Eq.(1) that

$$
P=(A+B+D) Q+C P-\frac{b Q}{(e P-d Q)},
$$

and

$$
Q=(A+B+D) P+C Q-\frac{b P}{(e Q-d P)} .
$$

Consequently, we get

$$
P+Q=\frac{b}{[e(1-C)-d(A+B+D)]},
$$

where $e(1-C)-d(A+B+D)>0$,

$$
P Q=\frac{e b^{2}(1-C)}{(e+d)\left[(1-C)+K_{4}\right]\left[e(1-C)-d K_{4}\right]^{2}},
$$

where $K_{4}=(A+B+D)$, provided $C<1$. Substituting (42) and (43) into (28), we get the condition (39). Thus, the proof is now completed.

\section{Boundedness of the solutions}

In this section, we investigate the boundedness of the positive solutions of Eq.(1).
Theorem 13.Let $\left\{x_{n}\right\}$ be a solution of Eq.(1). Then the following statements are true:

(i) Suppose $b<d$ and for some $N \geq 0$, the intial conditions

$$
x_{N-l+1}, \ldots, x_{N-k+1}, \ldots, x_{N-1}, x_{N} \in\left[\frac{b}{d}, 1\right],
$$

are valid, then for $b \neq e$ and $d^{2} \neq b e$, we have the inequality

$$
\frac{b}{d}(A+B+C+D)+\frac{b^{2}}{\left(d^{2}-b e\right)} \leq x_{n} \leq(A+B+C+D)+\frac{b}{(b-e)},
$$

for all $n \geq N$.

(ii) Suppose $b>d$ and for some $N \geq 0$, the intial conditions

$$
x_{N-l+1}, \ldots, x_{N-k+1}, \ldots, x_{N-1}, x_{N} \in\left[1, \frac{b}{d}\right],
$$

are valid, then for $b \neq e$ and $d^{2} \neq b e$, we have the inequality

$(A+B+C+D)+\frac{b}{(b-e)} \leq x_{n} \leq \frac{b}{d}(A+B+C+D)+\frac{b^{2}}{\left(d^{2}-b e\right)}$,

for all $n \geq N$.

Proof. First of all, if for some $N \geq 0, \frac{b}{d} \leq x_{N} \leq 1$ and $b \neq e$, we have

$$
\begin{aligned}
x_{N+1} & =A x_{N}+B x_{N-k}+C x_{N-l}+D x_{N-\sigma}+\frac{b x_{N-k}}{d x_{N-k}-e x_{N-l}} \\
& \leq A+B+C+D+\frac{b x_{N-k}}{d x_{N-k}-e x_{N-l}} .
\end{aligned}
$$

But, it is easy to see that $d x_{N-k}-e x_{N-l} \geq b-e$, then for $b \neq e$, we get

$$
x_{N+1} \leq A+B+C+D+\frac{b}{b-e} .
$$

Similarly, we can show that

$$
x_{N+1} \geq \frac{b}{d}(A+B+C+D)+\frac{b x_{N-k}}{d x_{N-k}-e x_{N-l}} .
$$

But, one can see that $d x_{N-k}-e x_{N-l} \leq \frac{d^{2}-b e}{d}$, then for $d^{2} \neq$ be, we get

$$
x_{N+1} \geq \frac{b}{d}(A+B+C+D)+\frac{b^{2}}{d^{2}-b e} .
$$

From (47) and (49) we deduce for all $n \geq N$ that the inequality (44) is valid. Hence, the proof of part (i) is completed.

Similarly, if $1 \leq x_{N} \leq \frac{b}{d}$, then we can prove part (ii) which is omitted here for convenience. Thus, the proof is now completed. $\square$ 


\section{Global stability}

In this section we study the global asymptotic stability of the positive solutions of Eq.(1).

Theorem 14.If $0<A+B+C+D<1$ and $e \neq d$, then the equilibrium point $\widetilde{x}$ given by (7) of Eq.(1) is global attractor.

Proof. We consider the following function

$$
F(x, y, z, w)=A x+B y+C z+D w+\frac{b y}{(d y-e z)},
$$

where $d y \neq e z$. It is easy to verify the condition (i) of Theorem 3. Let us now verify the condition (ii) of Theorem 3 as follows:

$$
\begin{aligned}
{[F(x, x, x, x)-x](x-\widetilde{x})=} & \left\{K_{5} x-\frac{b}{e-d}-x\right\} \\
& \times\left\{x-\frac{b}{\left[K_{5}-1\right](e-d)}\right\} \\
= & \left\{\frac{x(e-d)\left[K_{5}-1\right]-b}{e-d}\right\}^{2} \\
& \times \frac{1}{\left[K_{5}-1\right]},
\end{aligned}
$$

where $K_{5}=(A+B+C+D)$. Since $0<K_{5}<1$ and $e \neq d$, then we deduce from (51) that

$$
[F(x, x, x, x)-x](x-\widetilde{x})<0 .
$$

According to Theorem 3, $\widetilde{x}$ is global attractor. Thus, the proof is now completed. result.

On combining the two Theorems 4 and 14, we have the

Theorem 15.The equilibrium point $\widetilde{x}$ given by (7) of Eq.(1) is globally asymptotically stable.

\section{Numerical examples}

In order to illustrate the results of the previous section and to support our theoretical discussions, we consider some numerical examples in this section. These examples represent different types of qualitative behavior of solutions of Eq.(5).

Example 1. Figure 1, shows that Eq.(1) has no prime period two solutions if $k=2, l=4, \sigma=6, x_{-6}=$ $1, x_{-5}=2, x_{-4}=3, x_{-3}=4, x_{-2}=5, x_{-1}=6, x_{0}=7$, $A=300, B=200, C=100, D=75, b=50, d=$ $30, e=20$.

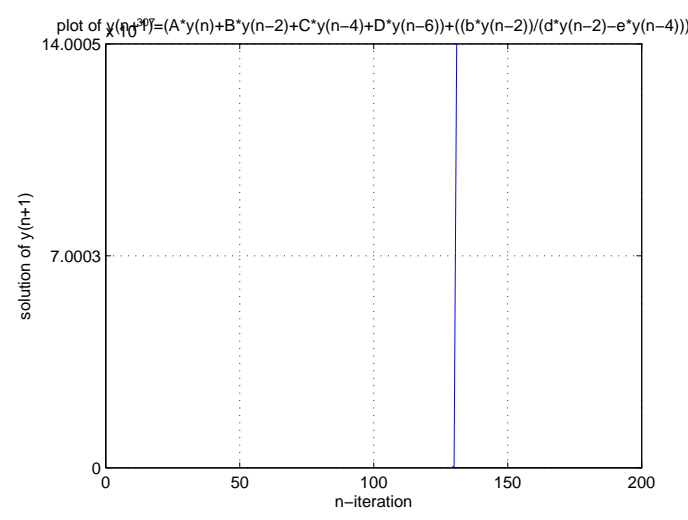

Example 2. Figure 2, shows that Eq.(1) has no prime period two solutions if $k=1, l=3, \sigma=5, x_{-5}=$ $1, x_{-4}=2, x_{-3}=3, x_{-2}=4, x_{-1}=5, x_{0}=6$, $A=75, B=50, C=25, D=20, b=5, d=3, e=2$.

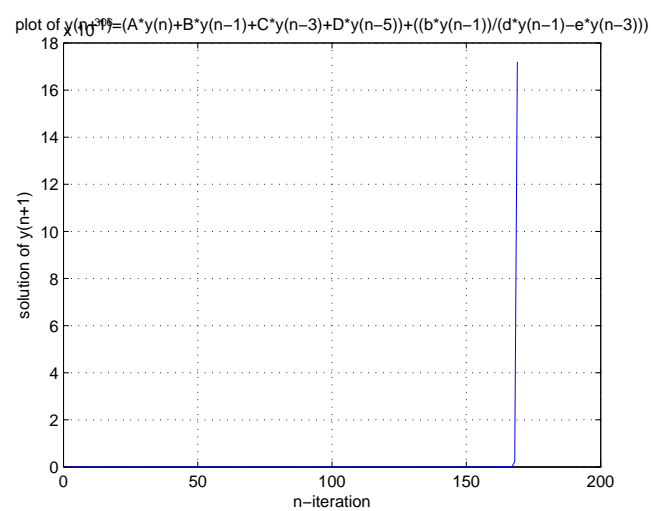

Example 3. Figure 3, shows that Eq.(1) has no prime period two solutions if $k=2, l=4, \sigma=5, x_{-5}=$ $1, x_{-4}=2, x_{-3}=3, x_{-2}=4, x_{-1}=5, x_{0}=6$, $A=10, B=20, C=30, D=40, b=5, d=3, e=2$.

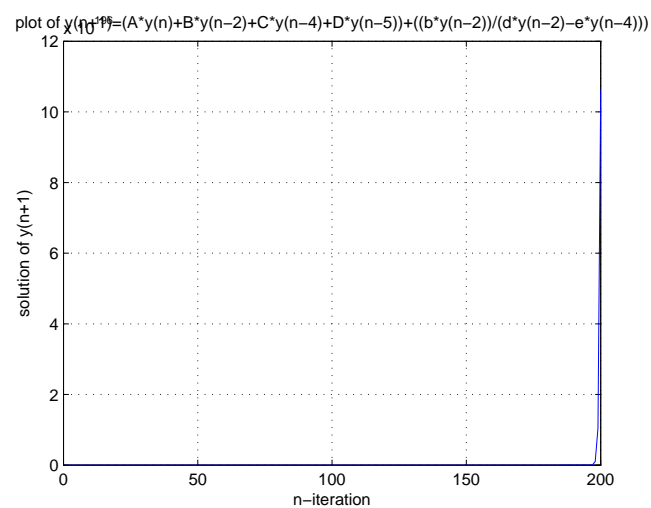


Example 4. Figure 4, shows that Eq.(1) has no prime period two solutions if $k=1, l=3, \sigma=4, \quad x_{-4}=$ $1, \quad x_{-3}=2, \quad x_{-2}=3, \quad x_{-1}=4, \quad x_{0}=5$, $A=100, B=50, C=25, D=200, b=5, d=3, e=2$.

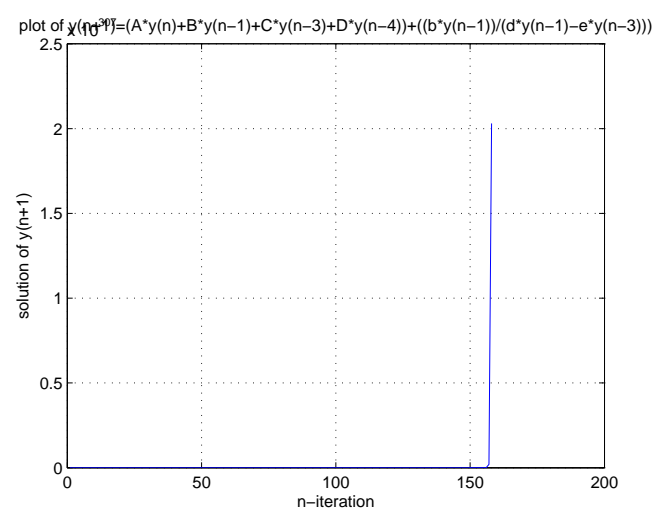

Example 5. shows that Eq.(1) is globally asymptotically stable if $k=1, l=3, \sigma=4, x_{-4}=$ $1, x_{-3}=2, x_{-2}=3, x_{-1}=4, x_{0}=5, A=0.01, B=$ $0.02, C=0.03, D=0.04, b=5, d=3, e=2000$.

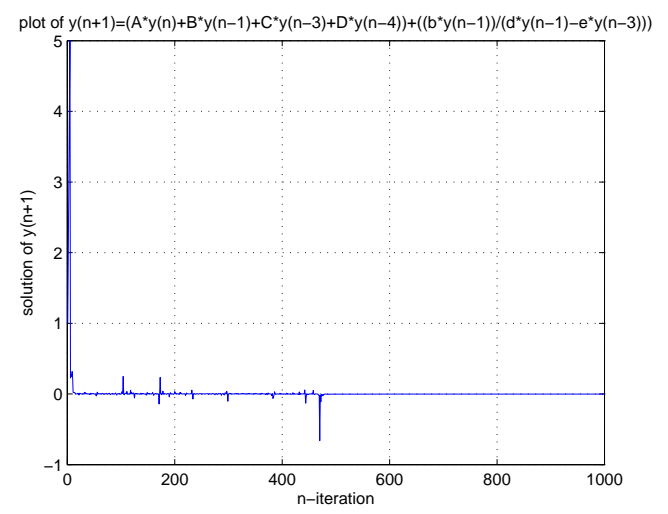

\section{Conclusion}

We have discussed some properties of the nonlinear rational difference equation (1), namely the periodicity, the boundedness and the global stability of the positive solutions of this equation. We gave some figures to illustrate the behavior of these solutions. The results in this article can be considered as a more generalization than the results obtained in Refs. [1,27,32,33]. We presented some numerical examples by giving some numerical values for the initial values and the coefficients of each case. Some figures have been given to explain the behavior of the obtained solutions in the case of numerical examples by using the mathematical programs Matlab and Mathematica to confirm the obtained results. Note that example 1 verifies Theorem 5 which shows that if $k, l$ and $\sigma$ are all even positive integers, then Eq.(1) has no prime period two solution and example 2 verifies Theorem 6 which shows that if $k, l$ and $\sigma$ are all odd positive integers, then Eq.(1) has no prime period two solution and example 3 verifies Theorem 7 which shows that if $k, l$ are even and $\sigma$ is odd positive integers, then Eq.(1) has no prime period two solution. But example 4 verifies Theorem 8 which shows that if $\sigma$ is even and $k, l$ are odd positive integers, then Eq.(1) has no prime period two solution, while example 5 verifies Theorem 15 which shows that Eq.(1) is globally asymptotically stable.

\section{References}

[1] E. M. Elabbasy, H. El- Metwally and E. M. Elsayed, On the difference equation $x_{n+1}=a x_{n}-b x_{n} /\left(c x_{n}-d x_{n-1}\right)$, Advances in Difference Equations, Volume 2006, Article ID 82579, pages 1-10, doi: 10.1155/2006/82579.

[2] H. El-Metwally and E. M. Elsayed, Solution and behavior of a third rational difference equation, Utilitas Mathematica, 88 (2012), 27-42.

[3] M. A. El-Moneam and S.O. Alamoudy, On study of the asymptotic behavior of some rational difference equations, DCDIS Series A: Mathematical Analysis (to appear).

[4] M. A. El-Moneam and E. M. E. Zayed, Dynamics of the rational difference equation, Inf. Sci. Lett. 3, No. 2, 1-9 (2014).

[5] E. M. Elsayed, Solution and attractivity for a rational recursive sequence, Discrete Dynamics in Nature and Society, Volume 2011, Article ID 982309, 17 pages.

[6] E. M. Elsayed, Solutions of rational difference system of order two, Mathematical and Computer Modelling, 55(2012), 378-384.

[7] E. M. Elsayed, Behavior and expression of the solutions of some rational difference equations, J. Computational Analysis and Applications, 15(2013), 73-81.

[8] E. M. Elsayed and M. M. El-Dessoky, Dynamics and behavior of a higher order rational recursive sequence, Advances in Difference Equations 2012, 2012:69 (28 May 2012).

[9] E. M. Elsayed and H. A. El-Metwally, On the solutions of some nonlinear systems of difference equations, Advances in Difference Equations 2013, 2013:16, doi:10.1186/16871847-2013-161, Published: 7 June 2013.

[10] M. E. Erdogan, C. Cinar and I. Yalcinkaya, On the dynamics of the recursive sequence, Computers \& Mathematics with Applications, 61(2011), 533-537.

[11] M. E. Erdogan, C. Cinar and I. Yalcinkaya, On the dynamics of the recursive sequence, Mathematical and Computer Modelling, 54(2011), 1481-1485.

[12] E. A. Grove and G. Ladas, Periodicities in nonlinear difference equations, Vol.4, Chapman \& Hall / CRC, 2005.

[13] T. F. Ibrahim, Solutions of a system of nonlinear fractional recursion equations, International Journal of Mathematical Education: Policy and Practice , 2(2012), 49-54. 
[14] T. F. Ibrahim, Periodicity and solution of rational recurrence relation of order six, Applied Mathematics , 3(2012), 729733.

[15] T. F. Ibrahim, Three-dimensional max-type cyclic system of difference equations, International Journal of Physical Sciences, 8(2013), 629-634.

[16] T. F. Ibrahim and N. Touafek, On a third-order rational difference equation with variable coefficients, DCDIS Series B: Applications \& Algorithms, 20(2013), 251-264.

[17] V. L. Kocic and G. Ladas, Global behavior of nonlinear difference equations of higher order with applications, Kluwer Academic Publishers, Dordrecht, 1993.

[18] M. R. S. Kulenovic and G. Ladas, Dynamics of second order rational difference equations with open problems and conjectures, Chapman \& Hall / CRC, Florida, 2001.

[19] N. Touafek and E. M. Elsayed, On the solutions of systems of rational difference equations, Mathematical and Computer Modelling, 55(2012), 1987-1997.

[20] N. Touafek and E. M. Elsayed, On the periodicity of some systems of nonlinear difference equations, Bull. Math. Soc. Sci. Math. Roumanie, Tome 55(103), No. 2, (2012), 217 224.

[21] I. Yalcinkaya and C. Cinar, On the dynamics of the difference equation $x_{n+1}=a x_{n-k} /\left(b+c x_{n}^{p}\right)$, Fasc. Math., 42 (2009), 141-148.

[22] E. M. E. Zayed, On the rational recursive sequence $x_{n+1}=$ $A x_{n}+B x_{n-k}+\left(1+x_{n} x_{n-k}\right) /\left(p x_{n}+x_{n-k}\right)$, Acta Math. Vietnamica, 37(2012), 251-266.

[23] E. M. E. Zayed and M. A. El-Moneam, On the rational recursive sequence $x_{n+1}=\left(D+\alpha x_{n}+\beta x_{n-1}+\right.$ $\left.\gamma x_{n-2}\right) /\left(A x_{n}+B x_{n-1}+C x_{n-2}\right)$, Comm. Appl. Nonl. Anal., 12(2005), 15-28.

[24] E. M. E. Zayed and M. A. El-Moneam, On the rational recursive sequence $x_{n+1}=\left(\alpha x_{n}+\beta x_{n-1}+\gamma x_{n-2}+\right.$ $\left.\delta x_{n-3}\right) /\left(A x_{n}+B x_{n-1}+C x_{n-2}+D x_{n-3}\right)$, J. Appl. Math. \& Computing, 22(2006), 247-262.

[25] E. M. E. Zayed and M. A. El-Moneam, On the rational recursive sequence $x_{n+1}=\left(A+\sum_{i=0}^{k} \alpha_{i} x_{n-i}\right) / \sum_{i=0}^{k} \beta_{i} x_{n-i}$, Math. Bohemica, 133(2008), No.3, 225-239.

[26] E. M. E. Zayed and M. A. El-Moneam, On the rational recursive sequence $x_{n+1}=$ $\left(A+\sum_{i=0}^{k} \alpha_{i} x_{n-i}\right) /\left(B+\sum_{i=0}^{k} \beta_{i} x_{n-i}\right)$, Int. J. Math. \& Math. Sci., Volume 2007, Article ID 23618, 12 pages, doi: 10.1155/2007/23618.

[27] E. M. E. Zayed and M. A. El-Moneam, On the rational recursive sequence $x_{n+1}=a x_{n}-b x_{n} /\left(c x_{n}-d x_{n-k}\right)$, Comm. Appl. Nonlinear Analysis, 15(2008), 47-57.

[28] E. M. E. Zayed and M. A. El-Moneam, On the Rational Recursive Sequence $x_{n+1}=\left(\alpha+\beta x_{n-k}\right) /\left(\gamma-x_{n}\right)$, J. Appl. Math. \& Computing, 31(2009) 229-237.

[29] E. M. E. Zayed and M. A. El-Moneam, On the rational recursive sequence $x_{n+1}=A x_{n}+$ $\left(\beta x_{n}+\gamma x_{n-k}\right) /\left(C x_{n}+D x_{n-k}\right), \quad$ Comm. Appl. Nonl. Anal., 16(2009), 91-106.

[30] E. M. E. Zayed and M. A. El-Moneam, On the Rational Recursive Sequence $x_{n+1}=\gamma x_{n-k}+$ $\left(a x_{n}+b x_{n-k}\right) /\left(c x_{n}-d x_{n-k}\right)$, Bull. Iranian Math. Soc., 36(2010) 103-115.

[31] E. M. E. Zayed and M. A. El-Moneam, On the rational recursive sequence $x_{n+1}=A x_{n}+B x_{n-k}+$ $\left(\beta x_{n}+\gamma x_{n-k}\right) /\left(C x_{n}+D x_{n-k}\right) \quad, \quad$ Acta Appl. Math., 111(2010), 287-301.
[32] E. M. E. Zayed and M. A. El-Moneam, On the rational recursive two sequences $x_{n+1}=a x_{n-k}+$ $b x_{n-k} /\left(c x_{n}+\delta d x_{n-k}\right)$, Acta Math. Vietnamica, 35(2010), 355-369.

[33] E. M. E. Zayed and M. A. El-Moneam, On the global attractivity of two nonlinear difference equations, J. Math. Sci., 177(2011), 487-499.

[34] E. M. E. Zayed and M. A. El-Moneam, On the rational recursive sequence $x_{n+1}=$ $\left(A+\alpha_{0} x_{n}+\alpha_{1} x_{n-\sigma}\right) /\left(B+\beta_{0} x_{n}+\beta_{1} x_{n-\tau}\right)$, Acta Math. Vietnamica, 36(2011), 73-87.

[35] E. M. E. Zayed and M. A. El-Moneam, On the rational recursive sequence $x_{n+1}=$ $\left(\alpha_{0} x_{n}+\alpha_{1} x_{n-l}+\alpha_{2} x_{n-k}\right) /\left(\beta_{0} x_{n}+\beta_{1} x_{n-l}+\beta_{2} x_{n-k}\right)$, Math. Bohemica, 135(2010), 319-336.

[36] E. M. E. Zayed and M. A. El-Moneam, On the global asymptotic stability for a rational recursive sequence, Iranian Journal of science and Technology (A: siences), 35(A4) (2011), 333-339.

[37] E. M. E. Zayed and M. A. El-Moneam, On the rational recursive sequence $x_{n+1}=\frac{\alpha_{0} x_{n}+\alpha_{1} x_{n-1}+\alpha_{2} x_{n-m}+\alpha_{3} x_{n-k}}{\beta_{0} x_{n}+\beta_{1} x_{n-1}+\beta_{2} x_{n-m}+\beta_{3} x_{n-k}}$, WSEAS Transactions Math., 11(2012), 373-382.

[38] E. M. E. Zayed and M. A. El-Moneam, On the qualitative study of the nonlinear difference equation $x_{n+1}=\frac{\alpha x_{n-\sigma}}{\beta+\gamma x_{n-\tau}^{p}}$, Fasciculi Mathematici, 50(2013), 137-147.

[39] E. M. E. Zayed and M. A. El-Moneam, Dynamics of the rational difference equation $x_{n+1}=\gamma x_{n}+\frac{\alpha x_{n-l}+\beta x_{n-k}}{A x_{n-l}+B x_{n-k}}$, Comm. Appl. Nonl. Anal., 21(2014), 43-53. 


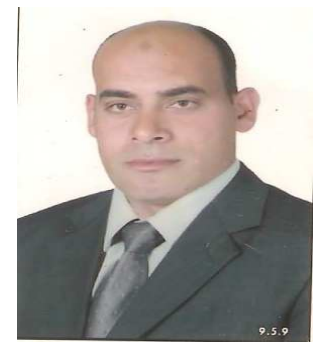

\section{A. El-Moneam}

Assistant Professor of Mathematics (from 2010 till now) at Mathematics Department, Faculty of Science and Arts in Farasan, Jazan University, Kingdom of Saudi Arabia. His interests are the nonlinear differential equations, Nonlinear difference equations. He Published many articles in famous International Journals around the world. He has reviewed many articles for many international Journals. He has got MSC degree in Mathematics from Faculty of Science, at Zagazig University, Egypt, supervised Prof. E. M. E. Zayed. He has got his PHD in Mathematics from Faculty of Science, at Zagazig University, Egypt, supervised Prof. E. M. E. Zayed. He has got the Man of the Year award, (2011), by the American Biographical, Institute, U.S.A. He is a member of International Society of Difference Equations (ISDE) in U.S.A. He is a member of peer reviewer in AIP Conference Proceeding 2010 Athena $(2010$, 2011, 2012, 2013). He is a member of peer reviewer in the 2nd Scientific Conference for students of higher education in Jedda, Kingdom of Saudi Arabia (1432 G.). He is a member of peer reviewer in the 3rd Scientific Conference for students of higher education in Al Khobar, Kingdom of Saudi Arabia 1433. He is a member of peer reviewer in the 4th Scientific Conference for students of higher education in Makkah, Kingdom of Saudi Arabia 1434. He is a member of peer reviewer in the 5th Scientific Conference for students of higher education in Riyadh, Kingdom of Saudi Arabia 1435. $\mathrm{He}$ is a peer reviewed to the Scientific research No. (164/662/1432), King Abd Al Aziz University, Kingdom of Saudi Arabia (1432 H.). He edited and reviewed the Scientific book No. (208), Taif University, Kingdom of Saudi Arabia, 2013 G., (1434 H. ). He has attended the Scientific sessions of the 4th international Exhibition and Conference on Higher Education (16-17/4/2013 G.), Riyadh, Kingdom of Saudi Arabia. He has attended the 1st Saudi Scientific Publishing Conference (27-29/3/1435 H.)(28-30/1/2014 G.) King Khalid University, Abha, Kingdom of Saudi Arabia. He is a peer reviewed to the Scientific research No. (LGP-35-4), King Abdulaziz City for Science and Technology (KACST), Kingdom of Saudi Arabia (1435 H.). 